\title{
Delayed Presentation of Ventricular Septal Rupture After Untreated Inferior Myocardial Infarction
}

\author{
Patel $\mathrm{AK}^{1 *}$, Heller $\mathrm{TM}^{1}$, McKeown $\mathrm{J}^{2}$, Phan $\mathrm{L}^{2}$ and Sharma $\mathrm{A}^{2}$ \\ ${ }^{1}$ Internal Medicine, Broward Health Medical Center, Fort Lauderdale, FL, USA \\ ${ }^{2}$ Cardiovascular Disease, Broward Health Medical Center, Fort Lauderdale, FL, USA
}

${ }^{\star}$ Corresponding author: Alexander K Patel, Internal Medicine, Broward Health Medical Center, 520 SE 5th Avenue Apt 2408 Fort Lauderdale, USA; E-mail: akpatel@browardhealth.org

Received: June 28, 2021; Accepted: July 06, 2021; Published: July 14, 2021

\begin{abstract}
Ventricular septal rupture is one of the most devastating complications of post myocardial infarction. The mortality rate of post myocardial infarction ventricular septal rupture increases significantly for each week when left untreated. We describe a case of a 62-year-old male who presented with progressively worsening shortness of breath and lower extremity edema three months after a myocardial infarction. The patient was subsequently found to have an anterior apical ventricular septal rupture with left to right shunting. Requiring surgical repair.
\end{abstract}

Keywords: Myocardial infarction, Ventricular septal rupture

\section{Introduction}

The most devastating complication of post myocardial infarction (MI) involves tearing or rupture of infarcted myocardial tissue. The clinical course differs depending on the site of the complication, which may involve the septum, free wall, or papillary muscles. Before the age of reperfusion therapy, post-MI ventricular septal rupture (VSR) occurred in $1 \%$ to $3 \%$ of patients with STEMI. With fibrinolytic intervention, the occurrence of VSR is approximately $0.2 \%$ to $0.34 \%$. Among those who have received reperfusion therapy, it occurs more commonly in those who received fibrinolytic therapy rather than percutaneous coronary intervention. In current times, patients not undergoing reperfusion therapy for an acute $\mathrm{MI}$ is a rare entity. For these patients, the first day after post-MI VSR is survived by approximately $75 \%$ of patients, the first week approximately $50 \%$, two weeks $30 \%$, and only $4-15 \%$ of patients survive the first month. Current guidelines recommend immediate operative intervention in patients with septal rupture, regardless of their clinical status. We present a case of an older gentleman who arrived approximately two months after initial MI whose chief complaint was progressively worsening dyspnea associated with lower extremity edema, and orthopnea. An EKG was obtained which illustrated Q waves in leads II, III, and aVF. Ultimately, he was found to have an anterior apical ventricular septal rupture with left-to-right shunt. The patient underwent repair with a bovine patch as well as coronary artery bypass grafting with the aorta to the posterior descending artery via reverse saphenous vein graft. Today's literature demonstrates that patients with a post infarct VSR have a significantly high mortality rate that is typically described over a onemonth time frame. Our case illustrates a patient who presented two months after an inferior MI with a post infarct VSR who underwent surgical intervention and had resolution of his presenting symptoms.

\section{Case Presentation}

A 62-year-old male with a past medical history of hypertension and dyslipidemia presented with the complaint of progressive worsening shortness of breath for over a month. Patient admitted to worsening shortness of breath with movement as well as lying flat and improving with sitting up. Also, the patient noted to have lower extremity edema for over a month. Of note, the patient stated that three months prior, while working, he began sweating profusely and had to stop working to sit down. He had a friend that worked in the fire department who performed an EKG supposedly showing no acute abnormalities. The patient ultimately went home after the EKG. His symptoms of shortness of breath and edema progressively worsened since this point in time. At presentation to the ER, the physical exam was consistent with volume overload and a holosystolic murmur was heard best at the left sternal border. EKG was performed showing sinus tachycardia, old inferior infarct with small Q-waves in leads III and aVF, left atrial enlargement, right axis deviation, and mild T-wave inversions. Additionally, there was poor R-wave progression from V1 through V5 suggestive of possible old anterior infarct. Labs revealed a BNP of 1255 and negative troponin. Echocardiogram showed an ejection fraction of $30 \%$, moderate septal, posterior, and lateral wall hypokinesis, and submitral left ventricular aneurysm with a $0.5 \mathrm{~cm}$ ventricular septal defect with left to right shunting. He underwent an elective cardiac catheterization revealing a ventricular septal defect, aneurysmal left ventricle, and occluded left circumflex artery (Figure 1).

Right heart catheterization pressures showed right ventricular systolic pressure of $62 \mathrm{mmHg}$ with end diastolic pressure of $24 \mathrm{mmHg}$, pulmonary artery pressure $62 / 25 \mathrm{mmHg}$ with mean of $41 \mathrm{mmHg}$, and right ventricle oxygen saturation of $69.1 \%$. A transesophageal 


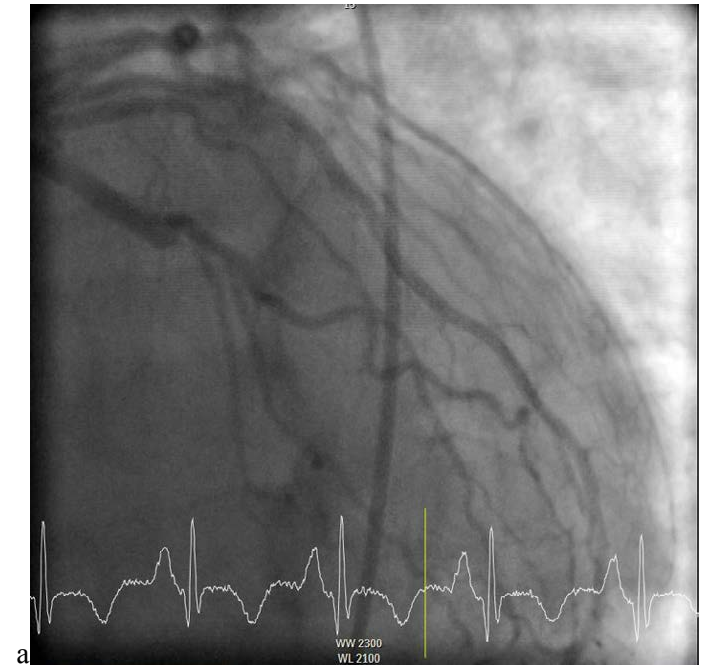

Figure 1: Left heart catheterization demonstrating an occluded left circumflex artery.

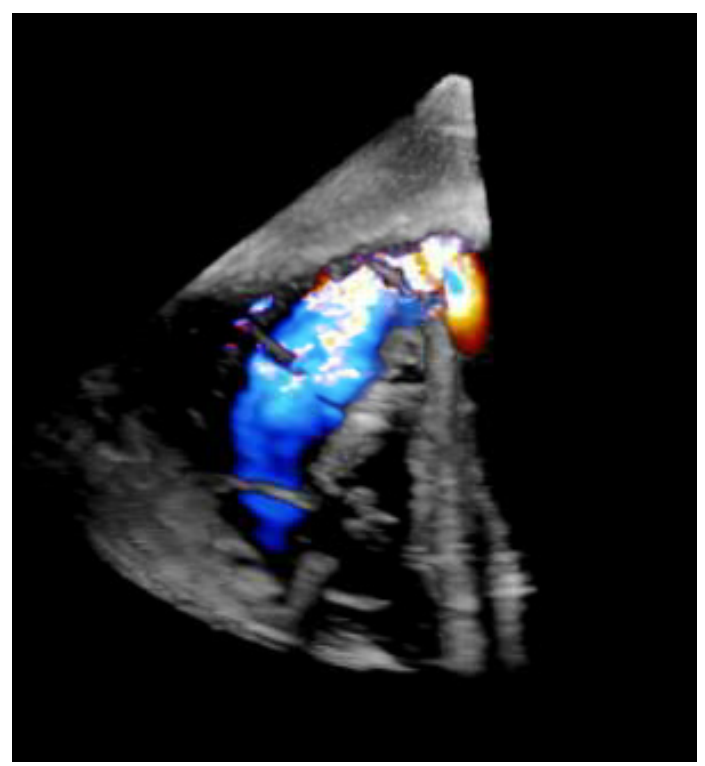

Figure 2: Short axis epigastric 3D TEE demonstrating ventricular septal rupture with left to right shunt. echocardiogram was completed due to concern for the ventricular septal defect being near the mitral valve, which would require replacement of the mitral valve during surgery. However, on TEE the ventricular septal defect was found to be apical with evidence of left to right shunting (Figures 2-4)

Ultimately, the patient underwent repair of the post infarct anterior apical ventricular septal defect with bovine patch pericardium, as well as a coronary artery bypass grafting from aorta to posterior descending artery with reverse saphenous vein graft. Patient did well in the postoperative period and was discharged in good condition. He followed up in the Cardiology clinic 1 month after discharge and denied complaints of chest pain, shortness of breath, and lower extremity edema.

\section{Discussion}

VSR is a rare, but devastating complication usually occurring within the first week of post-myocardial infarction. Only $0.17-0.31 \%$ of patients experience VSR due to modern reperfusion modalities, such as thrombolysis and primary percutaneous interventions [1]. Anterior infarction, advanced age, female sex, and no smoking history are factors most associated with VSR complicating acute myocardial infarction [2]. In addition, cardiogenic shock at the time of surgery as well as incomplete revascularization were found to be independent, strong predictors of poor 30-day, and long-term survival [3].

The blood flow to the septum is derived from branches of the left anterior descending artery and the posterior descending artery. Nearly two-thirds of VSR occur in the anterior septal wall, and about one-third in the inferior or posterior wall. Three mechanisms of rupture have been proposed by Becker. Type I is sudden in onset, within $24 \mathrm{hrs}$ of a myocardial infarction and is typically due to a dissecting intramural hematoma. These have been described in small inferior MI's that involve tissue associated with the distribution of the posterior descending artery. The primary mechanism for rupture is physical shear stressors, especially at the junction of the infarct area and normal healthy tissue receives blood supply from the left anterior descending artery [4]. Type II rupture involves the pathological

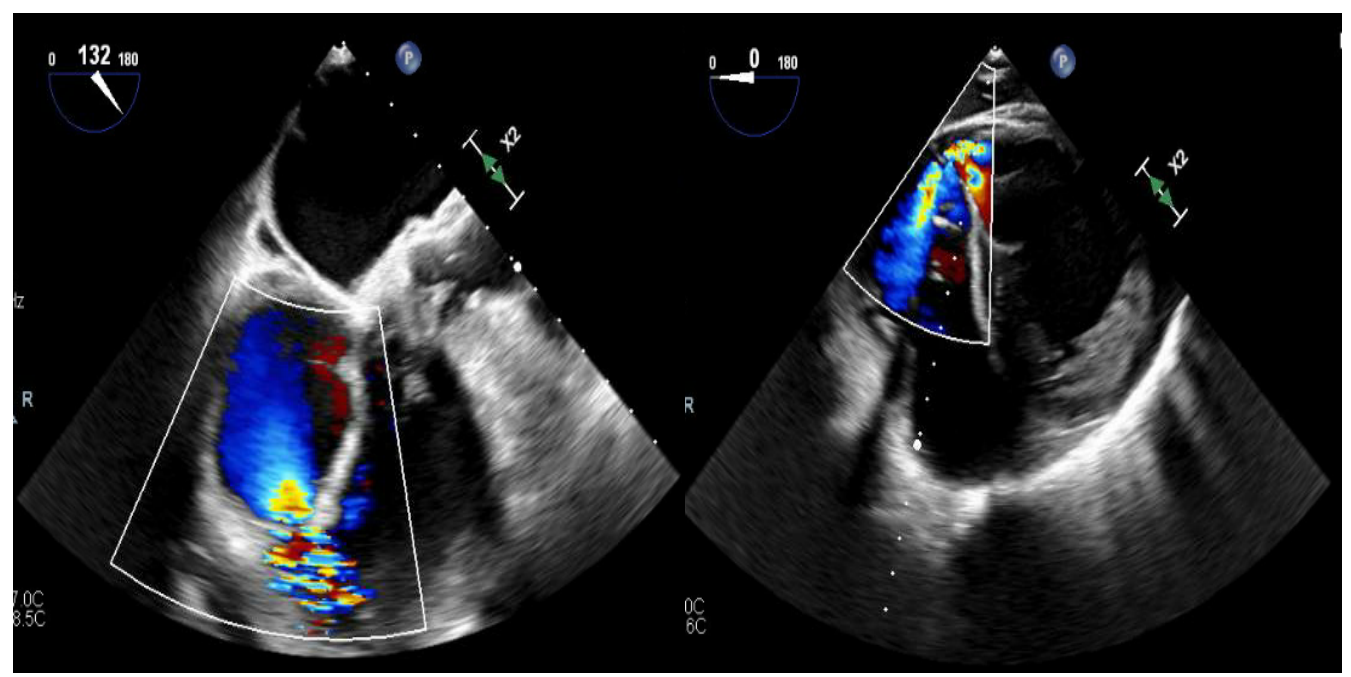

Figures 3 and 4: Figure on left illustrates a view of the right ventricle, left ventricle, and left ventricular outflow tract. Figure on the right illustrates a short axis epigastric view. These figures demonstrate a ventricular septal rupture with left to right shunting. 
finding of an infarcted septum and subsequent coagulation necrosis, which is a dry denaturation of proteins due to a lack of oxygen. Coagulation necrosis will progress to thinning and weakening of the septum, which takes approximately three to five days after an acute myocardial infarction, thus the presentation is typically subacute. Type III ruptures are more frequently encountered in patients that do not receive reperfusion therapy and occur due to perforation of a thinned, aneurysmal myocardial septum during the late post MI period [5].

The clinical presentation of VSR varies from asymptomatic murmur to advanced cardiogenic shock; however, a holosystolic murmur is heard in virtually all cases. Regarding diagnostic studies, echocardiography will likely demonstrate right ventricular dilatation and pulmonary hypertension due to the shunting of blood. When views are difficult or limited via a transthoracic echocardiogram, a transesophageal echocardiogram can be obtained. Cardiac catheterization in hemodynamically stable patients can illustrate a step up of oxygen between the right atrium and right ventricle and can help differential ventricular septal rupture from mitral regurgitation.

Acute treatment involves vasodilators to reduce afterload and potentially decrease the left to right shunting. However, in patients with low cardiac output, an intra-aortic balloon pump is vital for temporary hemodynamic support. Achieving hemodynamic stability prior to surgical treatment is beneficial; nevertheless, stabilization should not take priority over surgical repair, as this has been shown to have poor outcomes [6]. Medical therapy alone has a $90 \%$ mortality rate, and the current guidelines of the American College of Cardiology and American Heart Association recommend immediate surgical intervention regardless of the patient's hemodynamic status [7].
Our patient exhibited a post-myocardial infarction VSR with presentation 3 months after symptom onset. He had resolution of his symptoms and was hemodynamically stable after surgical intervention. Not only are post-infarct VSRs rare in today's era of reperfusion therapy, his survival, in a time frame with such high mortality rates, is what makes this case extremely unique. This case demonstrated that physicians should still carry a high suspicion for VSR as swift surgical intervention is crucial to increase survival of the deadliest complications of an MI.

\section{References}

1. Moreyra AE, Huang MS, Wilson AC, Deng Y, Cosgrove NM, et al. (2010) Trends in incidence and mortality rates of ventricular septal rupture during acute myocardial infarction. Am J Cardiol 106: 1095-1100. [crossref]

2. Crenshaw BS, Granger CB, Birnbaum Y, Pieper KS, Morris DC, et al. (2000) Risk factors, angiographic patterns, and outcomes in patients with ventricular septal defect complicating acute myocardial infarction. GUSTO-I (Global Utilization of Streptokinase and TPA for Occluded Coronary Arteries) Trial Investigators. Circulation 101: 27-32. [crossref]

3. Lundblad R, Abdelnoor M, Geiran OR, Svennevig JL (2009) Surgical repair of postinfarction ventricular septal rupture: risk factors of early and late death. J Thorac Cardiovasc Surg 137: 862-868. [crossref]

4. Mubarik A, Iqbal AM (2021) Ventricular Septal Rupture In: StatPearls [Internet]. Treasure Island (FL): StatPearls Publishing; 2021 Jan-. Available from: https://www. ncbi.nlm.nih.gov/books/nbk534857/

5. Goyal A, Menon V (2018) Contemporary Management of Post-MI Ventricular Septal Rupture. American College of Cardiology.

6. Heitmiller R, Jacobs ML, Daggett WM (1986) Surgical management of postinfarction ventricular septal rupture. Ann Thorac Surg 41: 683-691. [crossref]

7. Cannon CP, Brindis RG, Chaitman BR, et al. (2013) ACCF/AHA key data elements and definitions for measuring the clinical management and outcomes of patients with acute coronary syndromes and coronary artery disease: a report of the American College of Cardiology Foundation/American Heart Association Task Force on clinical data standards. J Am Coll Cardiol 61: 992-1025. [crossref] 